\title{
Articles
}

\section{Effects of Dimaine, Diacid and Dintitro Derivatives on the Inhibition of Adenosine Deaminase; Experimental, Molecular Docking and QSAR Studies}

\author{
Davood Ajloo, ${ }^{*}$ Leila Najafi, and Ali Akbar Saboury ${ }^{\dagger}$ \\ School of Chemistry, Damghan Linversity of Basic Science, Damghan, Iran. "E-mail: ajloodatubs.acir \\ ${ }^{\dagger}$ Institute of Biochemistry and Biophysics. The Chiversity of Tehran, Tehran. Iran \\ Received LAv 19, 2009, Accepted July 10, 2009
}

\begin{abstract}
Effects of some diacid, diamine and dinitro aromatic compounds on the structure and activity of adenosine deaminase (ADA) were investigated by UV-Vis spectrophotometry in $50 \mathrm{mM}$ phosphate buffer at $\mathrm{pH}=7.5$ and $27{ }^{\circ} \mathrm{C}$ and molecular docking studies. The results showed that all tested ligands are showing inhibition: five ligands are uncompetitive and other two ligands are mixed of competitive and noncompetetive inllibitors with majority of competitive behavior. For the later case analy sis was done based on competitive inhibition. Diacids have larger size and higher inhibition constant $(K)$ relative to others. A logical correlation between calculated free energy of binding and experimental values was obtained for un-connetitive. Experinental and calculated data showed that competitive inlibitors are distributed near the active site of enzyme and form several cluster of ranks, whereas uncompetitive inhibitors bind to the enzyme-substrate complex and distributed far from the active site. Results of structure-activity relationship showed that, larger, more hydrophobe, less spherical and more aromatic ligands have higher inhibition constants.
\end{abstract}

Key Words: Binding constant, QSAR. Enzyme inhibition, Principal conponent analy sis

\section{Introduction}

Adenosine deaminase (E.C.3.5.4.4) is a monomeric protein $(34.5 \mathrm{kDa})$. which catalyzes the deamination of adenosine and 2 '-deoxyadenosine nucleosides to their respective inosine derivative nucleosides and amunonia with a rate enhancement of $2 \times$ $10^{12}$ relative to the non-enzymatic reaction. ${ }^{1}$ The enzyme is present virtually in all human tissues. but the highest level is found in the lymphoid system such as lymph nodes, spleen, and thymus. ${ }^{2-4}$

The inhibition of adenosine deaminase in the brain would allow for an accumulation of adenosine. which would produce vasodilatation and increase in cerebral blood flow. Adenosine has come to be considered as an important factor in the attenuation of inflammation ${ }^{6 / 2}$ since it has been reported that the concentration of adenosine is increased in inflammatory lesions. ${ }^{8.11}$ Therefore. it is considered that an ADA inhibitor may prevent adenosine released specifically at inflamed sites from metabolism by ecto-ADA and would have great potential as an antiinflammatory drug with few side effects.

Effect of inosine. " caffeine. ${ }^{13}$ theophyline. ${ }^{14}$ acetaminophen, ${ }^{15}$ theobromine ${ }^{16}$ as inhibitors on ADA activity has been studied by spectroscopy and calorimetry. The enthalpy, equilibrium and inlibition constants for binding were obtained. ${ }^{1.18}$

On the other hand, performing QSAR analysis for several series of drugs, macromolecules enzy me and biological active compounds is now well appreciated. Kinetic parameters were used for the QSAR analysis and as such, we found some theoretical descriptors which correlated the binding affinity of ADA towards several adenine nucleosides as inhibitors. ${ }^{19}$ The kinetic parameters for adenosine deaminase were determined as well as the QSAR of these derivatives was studied. QSAR analysis revealed that the binding affinity depends on the molecular volume. dipole moment. electric charge, and the highest positive charge.

We previously investigated the effect of aspirin and diclofenac as non-steroid anti-inflammatory dnugs (NSAIDs) $)^{2(1)}$ on ADA using spectrophotometry and isothermal titration calorimetry. QSAR studies show that the large. polar, planar. and aromatic mucleoside and small, aromatic and polar non-nucleoside molecules have lower inhibition constant ${ }^{20}$

We have investigated effect of salts: solvents: and ionic surfactants on the structure and activity of adenosine deaninase (ADA) by UV-Vis spectrophotometery, circular dichroism and molecular dynamics (MD). ${ }^{21}$ Relative activity: experimental and computational helix content. total accessible surface area (ASA) and exposed charged surface area were obtained. It was shown that increasing the surface area and decreasing helix is associated to decreasing the activity.

The aim of this work was to study the effects of seven diacid. diamine and dinitro aromatic compounds on the activity of adenosine deaminase by UV-Vis spectrophotometry molecular docking and QSAR methods in order to more understanding the structure-activity relationship.

\section{Experimental}

Materials. Adenosine deaminase (type IV, from calf intestinal mucosa), were obtained from Roche.

Wholly aromatic ether-dianime (DAl) and wholly aromatic 
Scheme 1. Chemical structure and name of studied aromatic compounds in this work

Name

diether-diamine (DA2) were obtained from Merck and distilled in vacuum. Biphenyl-based diamine diacids (DACl and DAC2) synthesized same as literature. ${ }^{\text {?2 }}$ Imide containing aromatic diacid (DAC3) py ridine-based fully aromatic dinitro compounds (DN1, DN2) were synthesized as literature. ${ }^{2-7}$ The name. abbreviation and the structure of cited ligands were shown in Scheme 1. All of the other reagents were obtained from Merck chemical company.

\section{Methods}

Enzyme assay. Enzy'matic activities were assay'ed by UV-Vis spectrophotometry with a GBC 916 spectrophotometer. by following the decrease in absorbance at $265 \mathrm{~nm}$ resulting from the conversion of adenosine to inosine based on the Kaplan method. ${ }^{24}$ This method uses the change in the absorbance coefficient of adenosine $\left(\varepsilon=8400 \mathrm{M}^{-1} \mathrm{~cm}^{-1}\right)$ on conversion to inosine by the cataly tic activity of the enzyme. The adenosine concentration range was between $0.25 \sim 2.5$ times that of the $K_{\mathrm{m}}$. The concentration of enzyme in the assay mixture $(50 \mathrm{mM}$ sodium phosphate buffer. $\mathrm{pH} 7.5$ ) was $0.83 \mathrm{nM}$ with a final volume of $2 \mathrm{~mL}$. Activities were measured using at least five different concentrations of adenosine and the assays were performed at least in triplicate. Care was taken to use experimental conditions where the enzy'me reaction was linear during the first minutes of the reaction. It means that the linear range of $v$ versus [\$] substrate concentration curve was taken for data handling. This usually was carried out in the lower concentration range of substrate

Structure-activity relationship. Stnucture-activity analysis was performed on the cited conpounds with regarding to the following steps 1) entry of the molecular structures into adequate software to perform the structural optinization. 2) generation of the molecular descriptors. 3) statistical analysis through the multiple linear regression (MLR) and principal component analysis. In the first step. the molecular structure of different seven ligand inhibitors was constnucted and the three-dimensional structure optimized by the PM3 semi-empirical method in the Hyperchem-7.0 medium. In the second step. descriptors were generated using the Hyperchem-7.0 and Dragon-3.0 programs. ${ }^{25,26}$ Dragon can calculate 1497 descriptors in 18 classes such as topological. geometrical. empirical, constitutional, charge and so on. We select more biosence and interpretable descriptors. Some of descriptors may be correlated to each other. For the prevention of repeating descriptors and classifying them, we have used principal component analysis (PCA). PCA involves a mathematical procedure that transforms a number of (possibly) correlated variables into a (smaller) number of uncorrelated variables called principal components (PCs). Objectives of principal component analy sis are to, 1) Discover or reduce the dimensionality of the data set. 2) Identify' new meaningful underlying variables

This method reduces the descriptors into a few factors. Each factor describes a property same as geometrical, topological. electronic and lipophilic properties. These factors can be considered as a new descriptor that has same properties as original descriptors. For example size factor itself has property similar to molecular weight. surface area. number of atoms and number of functional group and so on.

Ligand docling. Version 3.0.5 of AutoDock ${ }^{2 ?}$ was used for the docking studies. ADA information was extracted from the protein databank (PDB) files of their X-ray crystal structures (IVFL) or optimized structure. Ligand PDB files were imported into Autodock tools (ADT). polar hydrogens were added. and Gasteiger charges were computed; the rigid root and the rotatable bonds were defined by the AutoTors tool of ADT. The PDB file was imported into ADT, all water molecules were removed. Kollman charges and solvation parameters were added. Grid maps of $126 \times 126 \times 126$ points with a grid-point spacing of $0.375 \AA$ were generated using the Auto Grid tool of $\mathrm{ADT}$. The 250 genetic algorithm $(\mathrm{GA})$ nuns were performed with the following parameters: population size of 50 . maximum number of $2.5 \times 10^{5}$ energy evaluations, maximum number of 27,000 generations, an elitism of 1 . a mutation rate of 0.02 , and a crossover rate of 0.8 . The resulting conformations were clustered using a root-mean-square deviation (rmsd) of $0.5 \mathrm{~A}$ and the clusters were ranked in order of increasing binding energy of the lowest binding energy conformation in each cluster. 


\section{Results and Discussion}

Enzy me kinetics. Schenc 1 shows 1he structure and nomenclature of seven compounds in three groups studied in this work. Dimaine (DAI and DA2), diacid (DACI. DAC2 and DAC3) and dinitro (DN1 and DN2) compounds lave two amine $\left(\mathrm{NH}_{2}\right)$. two acid $(\mathrm{COOH})$ and two nitro $\left(\mathrm{NO}_{2}\right)$ functional groups, res-
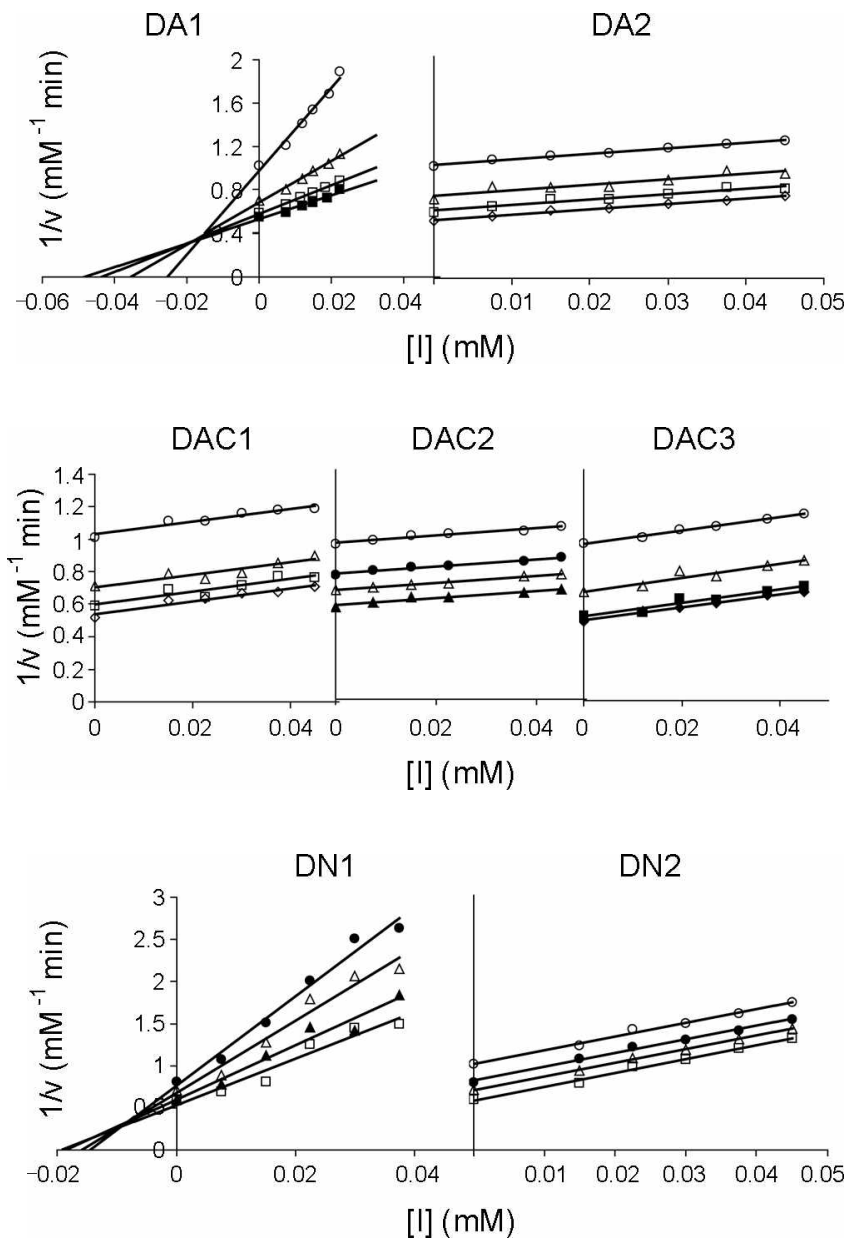

Figure 1. Lickson plot ( $1 / v$ agrinst inhibitor concentration, |II) lor ADA in the absence $(x)$ and presence ol $0.015(>), 0.0225(\bullet) 0.030$

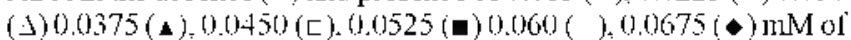
ademosine. pecively.

Fig. I shows Dickson plot ( $1 / \mathrm{k}$ is. concentration of inllibitor. [I]) in different fixed concent ration of adenosine and organic ligands at $50 \mathrm{mM}$ phospliate buffer. $\mathrm{pH} 7.5$ and $27^{\circ} \mathrm{C}$. Michiels constant $\left(K_{\mathrm{m}}\right)$ and maximum velocity ( $\left(_{\text {thix }}\right)$ are $26.8 \mu \mathrm{M}$ and. $2.73 \mathrm{mM} \mathrm{min}{ }^{-3}$. respectively. Fig. 1 also shows that two ligands. DAl and DNI, are mixed of competitive and non competitive inhibitors. They bind to the active site of enzyme. compete with substrate. ${ }^{*}$ The inhibition constant can be obtained from slope of $1 / \mathrm{k}$ w. [I] according to cquation (1).

$$
\frac{l}{v}=\frac{1}{v_{\mathrm{del}: \mathrm{K}} K_{J}^{\prime}}\lceil I\rceil+\frac{1}{v_{\max }}\left(1+\frac{K_{m}}{[S]}\right)
$$

DAI and DNI have smaller size and nore fav orable binding to active site than others do. In addition. based on Fig. 1. the other five ligands are uncompetitive inlibitors. Uncompetitive inhibitors bind to the enzyme-substrate (ES) complex. The inhibition constant $\left(\Lambda_{1}\right)$ for uncompetitive inhibitors can be obtained from slope of Dickson plot (1/v v. [I]) by cquation (2):

$$
\frac{l}{v}=\frac{K_{m}}{v_{\max } \Lambda_{J}\lfloor S]}[I\rfloor+\frac{1}{v_{\max }}\left(1+\frac{K_{m}}{[S]}\right)
$$

The values of $\overline{\mathrm{T}}_{\mathrm{T}}$. for DA1. DA2, DAC 1 , DAC2, DAC 3 . DN 1 and DN2 are 15.24. 78.70.97.05. 131.83. 79.61. 7.30 and $22.23 \mu \mathrm{M}$. respectively. DAC ligands have higher $K_{\mathrm{T}}$ relative to others.

Molecular docking. In order to clarifying differences between kinctic data for DA I. DN1 and ol her ligands. we used docking results. In the first step, structure of $\mathrm{ADA}$ was optimized up to 6000 ps in water by GROMACS molecular dy namics soltware. Secondly: docking of seven ligands to optimized ADA was studied by Autodock 3,0.5. The number of ruls was 250 and output of docking was sorted based on binding or docking free energy $\left(\Delta G_{1}\right)$. Most negative $K K_{1}$, located in the first of data scries. Table 1 listed the most ten negative. I $\xi_{\mathrm{l}}$. Relative. ( $\mathrm{f}_{\mathrm{h}}$ for ligands are DAC $3<$ DN $2<$ DA $1<$ DAC $1<$ DA $2<$ DNI $<$ DAC2. Fig. 2 illustrated distribution of docking sites for the first 10 ranks of docking belong to seven ligands to $\mathrm{ADA}$.

\begin{tabular}{|c|c|c|c|c|c|c|c|c|c|c|c|c|c|c|}
\hline \multirow{2}{*}{ Rank } & \multicolumn{7}{|c|}{$\backslash(\mathrm{r} / \mathrm{kcal} / \mathrm{mol}$} & \multicolumn{7}{|c|}{ Number of run in al clusiler } \\
\hline & DAI & DA2 & DACI & $\mathrm{DAC} 2$ & $\mathrm{DAC} 3$ & DNI & DN2 & $\mathrm{DAl}$ & DA2 & D)ACl & $\mathrm{DAC} 2$ & DAC3 & DNl & LN2 \\
\hline 1 & -7.70 & -9.92 & -9.27 & -6.12 & -11.84 & -10.68 & -11.35 & 6 & 2 & I & I & l & 2 & 1 \\
\hline 2 & -7.01 & -9.67 & -8.10 & -6.06 & -11.66 & -10.49 & -11.31 & 12 & 1 & 1 & 1 & 1 & 2 & 1 \\
\hline 3 & -7.12 & -9.5 .3 & -7.95 & -6.05 & -11.3 .3 & -10.51 & -11.06 & 6 & 1 & 1 & 1 & 1 & 1 & 1 \\
\hline 4 & -7.06 & -9.51 & -7.93 & -5.94 & -11.12 & -10.39 & -10.26 & 2 & I & 1 & I & 1 & I & 1 \\
\hline 5 & -7.60 & -9.44 & -7.75 & -5.57 & -11.11 & -9.89 & -10.1 & 2 & 1 & 1 & 1 & 1 & 2 & 1 \\
\hline 6 & -6.88 & -8.75 & -7.71 & -5.49 & & -9.73 & -10.05 & 5 & 3 & 1 & 1 & 1 & 8 & 1 \\
\hline 7 & -6.93 & -9.40 & -7.67 & -5.49 & -11.01 & -9.80 & -9.79 & 2 & 1 & 1 & 1 & 1 & 1 & 5 \\
\hline 8 & -6.97 & -9.30 & -7.54 & -5.47 & -10.97 & -9.72 & -9.77 & 1 & l & 1 & 1 & 1 & l & 6 \\
\hline$y$ & -6.77 & -9.24 & -7.51 & -5.30 & -10.84 & -9.60 & -9.96 & 1 & l & 1 & l & 1 & l & 1 \\
\hline 10 & -6.75 & -9.17 & -7.36 & -5.28 & -10.81 & -9.35 & -9.75 & 1 & l & 1 & l & 1 & 3 & 2 \\
\hline
\end{tabular}

Table 1. Free energy of binding and number of runs in a cluster for the first ten ranks of ligand binding to optimized $A D A$ 
Table 2. Symbol, definition and classification of used 43 descriptors

\begin{tabular}{|c|c|c|c|}
\hline No & symbol & Definition & class \\
\hline 1 & MW & molecular weight & constitutional descriptors \\
\hline 2 & AMW & average molecular weight & constitutional descriptors \\
\hline 3 & Sv & sum of atomic van der Waals volumes(scaled on Carbon atom) & constitutional descriptors \\
\hline 4 & $\mathrm{Se}$ & sum of atomic Sanderson electronegativities (scaled on Carbon atom) & constitutional descriptors \\
\hline 5 & $\mathrm{Sp}$ & sum of atomic polarizabilities ( scaled on Carbon atom) & constitutional descriptors \\
\hline 6 & Ss & sum of Kier-Hall electrotopological states & constitutional descriptors \\
\hline 7 & $\mathrm{Mv}$ & mean atomic van der Waals volume (scaled on Carbon atom) & constitutional descriptors \\
\hline 8 & $\mathrm{Me}$ & mean atomic Sanderson electronegativity (scaled on Carbon atom) & constitutional descriptors \\
\hline 9 & $\mathrm{Mp}$ & mean atomic polarizability (scaled on Carbon atom) & constitutional descriptors \\
\hline 10 & Ms & mean electrotopological state & constitutional descriptors \\
\hline 11 & $11 \mathrm{AT}$ & number of atoms & constitutional descriptors \\
\hline 12 & $\mathrm{nSK}$ & number of non- $\mathrm{H}$ atoms & constitutional descriptors \\
\hline 13 & $11 \mathrm{BT}$ & number of bonds & constitutional descriptors \\
\hline 14 & $\mathrm{nBO}$ & number of non- $\mathrm{H}$ bonds & constitutional descriptors \\
\hline 15 & $11 \mathrm{BM}$ & number of multiple bonds & constitutional descriptors \\
\hline 16 & SCBO & sum of conventional bond orders (H-depleted) & constitutional descriptors \\
\hline 17 & $11 \mathrm{CIC}$ & number of rings & constitutional descriptors \\
\hline 18 & nCIR & number of circuits & constitutional descriptors \\
\hline 19 & $\mathrm{RBN}$ & number of rotatable bonds & constitutional descriptors \\
\hline 20 & $\mathrm{RBF}$ & rotatable bond fraction & constitutional descriptors \\
\hline 21 & $12 \mathrm{DB}$ & number of donble bonds & constitutional descriptors \\
\hline 22 & $\mathrm{nAB}$ & number of aromatic bonds & constitutional descriptors \\
\hline 23 & $11 \mathrm{H}$ & number of Hydrogen atoms & constitutional descriptors \\
\hline 24 & $\mathrm{nC}$ & number of Carbon atoms & constitutional descriptors \\
\hline 25 & ${ }_{110}$ & number of Oxygen atoms & constitutional descriptors \\
\hline 26 & nR06 & number of 6 -membered rings & constitutional descriptors \\
\hline 27 & ${ }_{11} \mathrm{Bnnz}$ & number of benzene-like rungs & constitutional descriptors \\
\hline 28 & $\mathrm{Ui}$ & unsaturation index & empirical descriptors \\
\hline 29 & $\mathrm{Hy}$ & hydrophilic factor & empirical descriptors \\
\hline 30 & $\dot{M R}$ & Ghose-Crippen molar refractivity & properties \\
\hline 31 & PSA & fragment-based polar surface area & properties \\
\hline 32 & MLOGP & Moriguchi octanol-water partition coeff. (logP) & properties \\
\hline 33 & $\mathrm{HE}$ & hydration energy & properties $^{\sigma}$ \\
\hline 34 & AROM & aromaticity (trial) & aromaticity indices \\
\hline 35 & HOMT & HOMA total (trial) & aromaticity indices \\
\hline 36 & SPH & Spherosity & geometrical descriptors \\
\hline 37 & ASP & Asphericity & geometrical descriptors \\
\hline 38 & FDI & folding degree index & geometrical descriptors \\
\hline 39 & $\mathrm{~L} / \mathrm{Bw}$ & Length-to-breadth ratio by WHM & geometrical descriptors \\
\hline 40 & Mus & Dipole moment in s-direction & electrotopological ${ }^{\prime \prime}$ \\
\hline 41 & Muy & Dipole moment in y-direction & electrotopological ${ }^{a}$ \\
\hline 42 & Muz & Dipole monnent in z-direction & electrotopological" \\
\hline 43 & Mutot & total dipole moment & electrotopological ${ }^{n}$ \\
\hline
\end{tabular}

"Calculated by Hyperchem.

Stmicture-activity relationship. The 43 molecular properties (descriptors) were calculated using Dragon-3.0 and Hyperchem7.0 softwares. Table 2 includes name and definition of these descriptors which classified as constitutional ( $1-27)$. empirical (28-29). properties (30-33). aromaticity (34-35). geometrical (36-39) and electrotopology $(40-43)$.

The experimental $\log K_{\mathrm{I}}$ and calculated values of descriptors were listed in Table 3. Linear correlation $(R)$ between the $\log K_{\mathrm{I}}$ and these calculated parameters (descriptors), for total $(R 7)$ and uncompetitive ( $R 5)$ inhibitors were reported in the last two rows of this Table. Correlation between size and inhibition shows that larger size inhibitors have higher inhibition constant. Thus diacids have larger size and higher inlubition constant. Among diacids, DAC3 has smaller size and lower $K_{I}^{r}$ relative to others based on Table 3. Trend of size for diacids is DAC $3<\mathrm{DACl}<$ $\mathrm{DAC} 2$ and trend of $\log K_{\mathrm{I}}$ is also DAC $3<\mathrm{DAC} 1<\mathrm{DAC} 2$.

In order to clarifying correlation between $\log K_{\mathrm{I}}$ and descriptors, Fig. 3 was plotted. Fig. 3a shows majority of size dependent desciptors have positive correlation with $\log K_{\mathrm{I}}$, while some of them such as Hydration energy ( $\mathrm{HE}$ ) spherocity ( $\mathrm{SPH}$ ), folding degree index and dipole moment have negative correlation. Fig. 3b shows difference between descriptor values of a compe- 

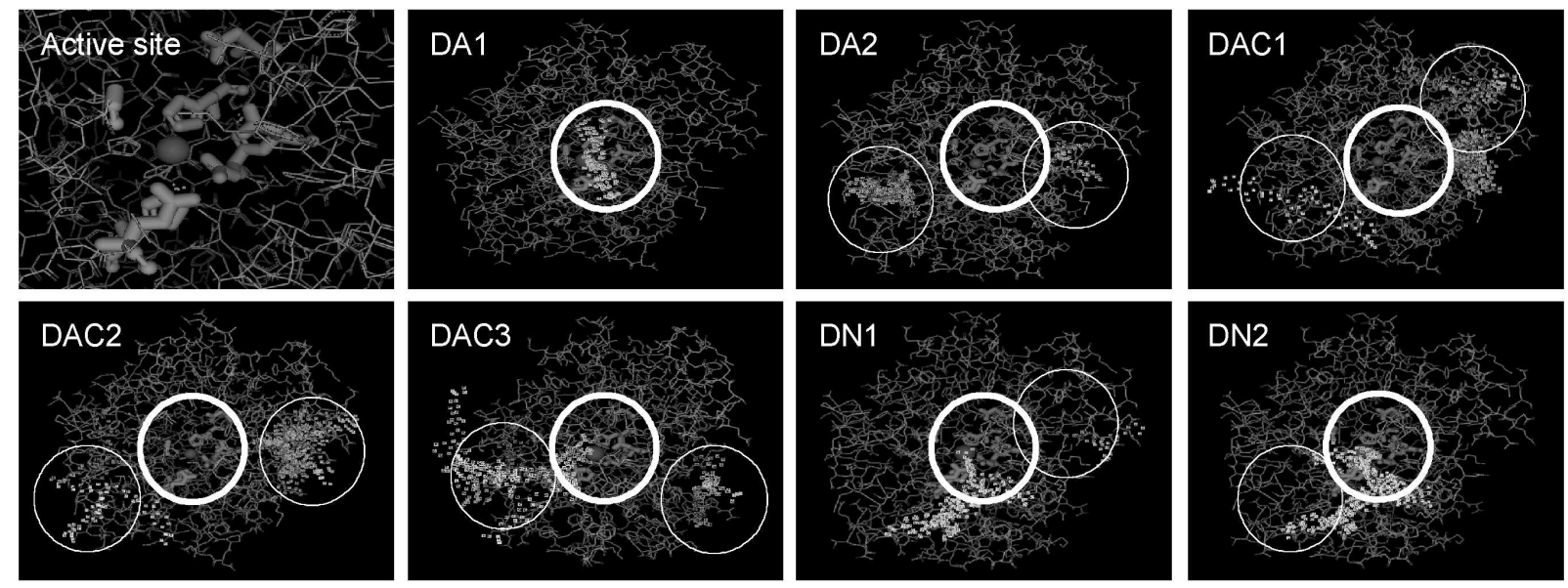

Figure 2. Snapshot of ligand distribution on the different sites of ADA extracted by Autodock 3.0.3. ADA optimized after $6000 \mathrm{ps}$ by GROMACS 3.3 molecular dynamics. Yellow, green and pink color, represent the ligand molecules, active site amino acid and \% atom, respectively.

Table 3. The values of experimental log $k_{1}$ and calculated descriptors for studied ligands

\begin{tabular}{|c|c|c|c|c|c|c|c|c|c|c|c|c|c|c|}
\hline Ligand & $\log K_{\mathrm{I}}$ & mass & AMW & Sv & $\mathrm{Se}$ & $S p$ & $\mathrm{Ss}$ & $\mathrm{Mv}$ & $\mathrm{Me}$ & $\mathrm{Mp}$ & Ms & nAT & nSK & $\mathrm{nBT}$ \\
\hline $\mathrm{DA}_{1}$ & 1.18 & 200.2 & 7.42 & 17.49 & 27.0 & 18.2 & 34.17 & 0.65 & 1.00 & 0.68 & 2.28 & 27 & 15 & 28 \\
\hline $\mathrm{D} \Lambda_{2}$ & 1.90 & 368.4 & 7.68 & 32.39 & 47.9 & 33.7 & 60.33 & 0.67 & 1.00 & 0.70 & 2.15 & 48 & 28 & 51 \\
\hline $\mathrm{DAC}$ & 1.99 & 716.7 & 9.19 & 55.68 & 80.3 & 56.9 & 142.3 & 0.71 & 1.03 & 0.73 & 2.64 & 78 & 54 & 85 \\
\hline $\mathrm{DAC}=$ & 2.12 & 816.8 & 9.08 & 64.88 & 92.1 & 66.4 & 157.0 & 0.72 & 1.02 & 0.74 & 2.53 & 90 & 62 & 99 \\
\hline $\mathrm{D} A \mathrm{C}_{3}$ & 1.90 & 685.7 & 9.14 & 54.05 & 76.8 & 55.2 & 136.3 & 0.72 & 1.02 & 0.74 & 2.62 & 75 & 52 & 82 \\
\hline$D V_{1}$ & 0.86 & 397.4 & 8.83 & 31.62 & 46.0 & 32.4 & 78.33 & 0.70 & 1.02 & 0.72 & 2.61 & 45 & 30 & 48 \\
\hline \multirow[t]{3}{*}{ D. $V_{2}$} & 1.35 & 473.5 & 8.61 & 38.81 & 55.8 & 39.9 & 89.67 & 0.71 & 1.01 & 0.73 & 2.49 & 55 & 36 & 59 \\
\hline & R7 & 0.74 & 0.33 & 0.76 & 0.77 & 0.77 & 0.69 & 0.39 & 0.29 & 0.44 & 0.03 & 0.78 & 0.74 & 0.78 \\
\hline & $\mathrm{R} 5$ & 0.60 & 0.25 & 0.62 & 0.64 & 0.63 & 0.55 & 0.05 & 0.41 & 0.09 & 0.09 & 0.65 & 0.60 & 0.65 \\
\hline
\end{tabular}

Table 3. continued

\begin{tabular}{cccccccccccccccc}
\hline nBO & nBM & SCBO & nCIC & nCIR & RBN & RBF & nDB & nAB & nH & Nc & nO & nR06 & nBnz & Ui & Hy \\
16 & 12 & 22 & 2 & 2 & 4 & 0.14 & 0 & 12 & 12 & 12 & 1 & 2 & 2 & 3.70 & 2.17 \\
31 & 24 & 43 & 4 & 4 & 6 & 0.12 & 0 & 24 & 20 & 24 & 2 & 4 & 4 & 4.64 & 1.56 \\
61 & 42 & 85 & 8 & 10 & 8 & 0.09 & 6 & 36 & 24 & 42 & 10 & 6 & 6 & 5.43 & -0.80 \\
71 & 52 & 100 & 10 & 14 & 8 & 0.08 & 6 & 46 & 28 & 50 & 10 & 8 & 8 & 5.73 & -0.80 \\
59 & 42 & 83 & 8 & 10 & 4 & 0.05 & 6 & 36 & 23 & 41 & 8 & 6 & 5 & 5.43 & -0.80 \\
33 & 28 & 47 & 4 & 4 & 2 & 0.04 & 1 & 27 & 15 & 23 & 4 & 4 & 3 & 4.86 & -0.80 \\
40 & 34 & 58 & 5 & 5 & 2 & 0.03 & 2 & 32 & 19 & 29 & 4 & 5 & 4 & 5.13 & -0.80 \\
0.74 & 0.67 & 0.72 & 0.77 & 0.77 & 0.84 & 0.20 & 0.69 & 0.64 & 0.88 & 0.77 & 0.67 & 0.72 & 0.82 & 0.61 & -0.20 \\
$\ldots .60$ & 0.47 & 0.57 & 0.64 & 0.68 & 0.89 & 0.65 & 0.52 & 0.44 & 0.80 & 0.63 & 0.59 & 0.54 & 0.71 & 0.40 & 0.10
\end{tabular}

Table 3. continued

\begin{tabular}{rrrrrrrrrrrrrr}
\hline MR & PSA & MLOGP & HE & HOMT & AROM & SPH & ASP & FDl & LiBw & mux & muy & muz & mutot \\
\hline 61.7 & 9.2 & 2.21 & -13.7 & 11.70 & 1.00 & 0.89 & 0.63 & 0.97 & 8.7 & 1.24 & 0.29 & -1.49 & 1.96 \\
113.1 & 18.5 & 3.81 & -14.0 & 23.47 & 1.00 & 0.51 & 0.25 & 0.91 & 3.5 & 0.39 & -0.25 & -0.97 & 1.07 \\
186.3 & 129.7 & 3.24 & -19.3 & 34.52 & 0.99 & 0.88 & 0.56 & 0.96 & 5.8 & -1.40 & -3.82 & -2.44 & 4.74 \\
219.2 & 129.7 & 4.62 & -18.8 & 38.93 & 0.95 & 0.78 & 0.48 & 0.93 & 5.1 & -1.68 & -4.11 & -3.69 & 5.77 \\
181.2 & 124.1 & 3.79 & -17.7 & 34.57 & 0.98 & 0.97 & 0.47 & 1.00 & 3.5 & 2.65 & -1.53 & -0.91 & 3.19 \\
113.4 & 93.5 & 4.76 & -14.8 & 26.00 & 0.61 & 1.00 & 0.29 & 1.00 & 1.6 & 0.18 & 8.01 & 0.00 & 8.01 \\
138.7 & 105.9 & 5.75 & -15.7 & 31.03 & 0.76 & 1.00 & 0.29 & 1.00 & 1.6 & 0.19 & 8.48 & 0.00 & 8.48 \\
\hline 0.74 & 0.35 & -0.11 & -0.77 & 0.62 & 0.73 & -0.51 & 0.21 & -0.60 & 0.19 & -0.29 & -0.83 & -0.70 & -0.39 \\
0.59 & 0.11 & -0.73 & -0.62 & 0.35 & 0.89 & -0.43 & 0.60 & -0.60 & 0.89 & -0.29 & -0.99 & -0.82 & -0.57 \\
\hline
\end{tabular}


(a)

(b)
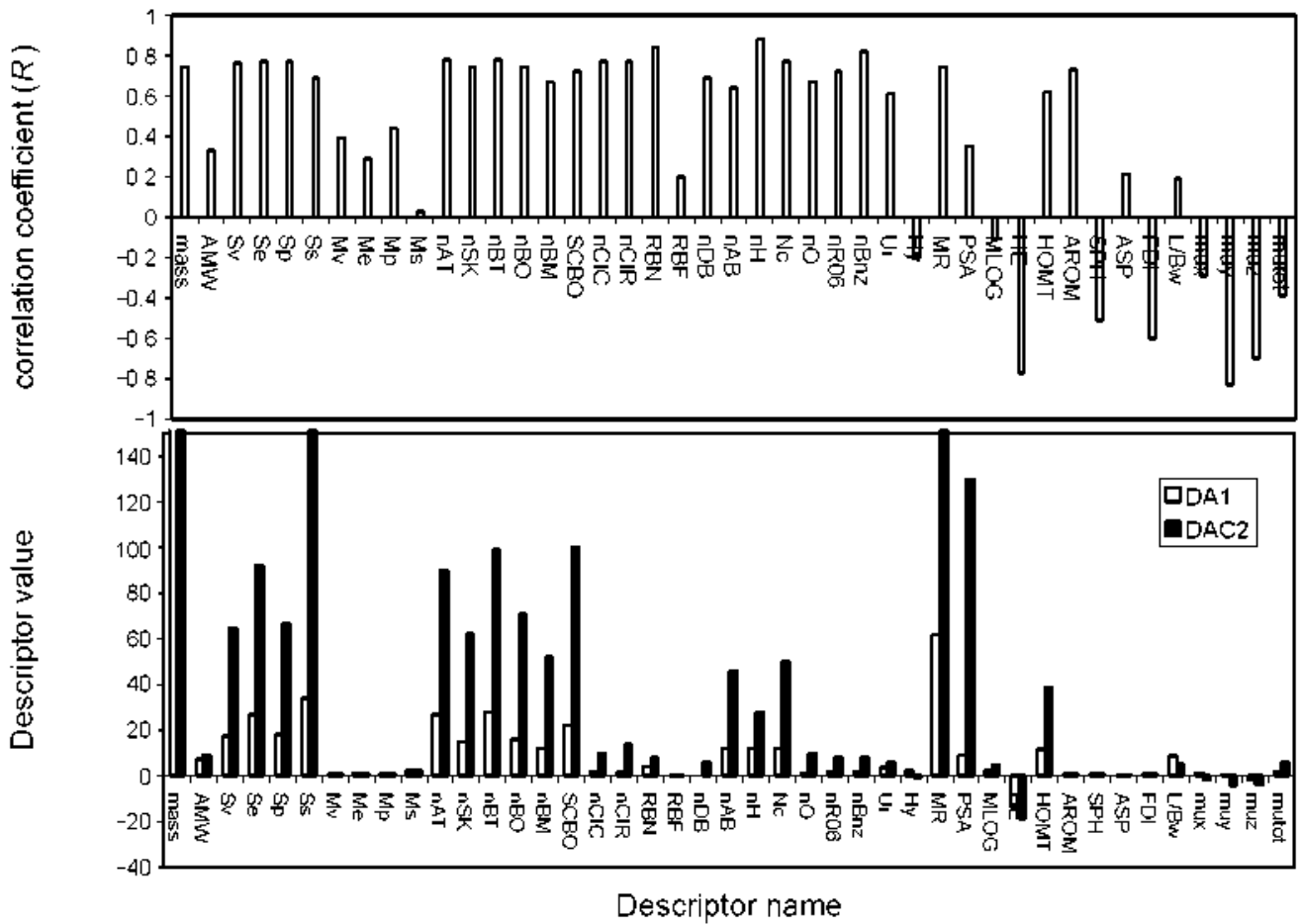

Figure 3. (a) correlation coefficient values between $\log K_{\text {l }}$ and descriptors (b) comparison between descriptor values of a competitive (DAl) and uncompetitive (DAC2) inhibitors. Scale of $y$ axis for simplicity in comparison only was shown up to 150.

Table 4. Total variance explained by two principal components or PCs

\begin{tabular}{|c|c|c|c|c|c|c|c|c|}
\hline \multirow[t]{2}{*}{ Component } & \multicolumn{3}{|c|}{ Initial Eigenvalues } & \multicolumn{3}{|c|}{ Extraction Sums of Squared Loadings } & \multicolumn{2}{|c|}{$\begin{array}{l}\text { Rotation Sums of } \\
\text { Squared Loadings }\end{array}$} \\
\hline & Total & $\%$ of Variance & Cumulative \% & Total & Cumulative \% & Total & $\%$ of Variance & Cumulative $\%$ \\
\hline $\mathrm{PCl}$ & 29.59 & 68.82 & 68.82 & 29.59 & 68.82 & 29.47 & 68.54 & 68.54 \\
\hline $\mathrm{PC} 2$ & 8.43 & 19.60 & 88.42 & 8.43 & 88.42 & 8.55 & 19.87 & 88.42 \\
\hline
\end{tabular}

Extraction Method: Principal Component Analysis.
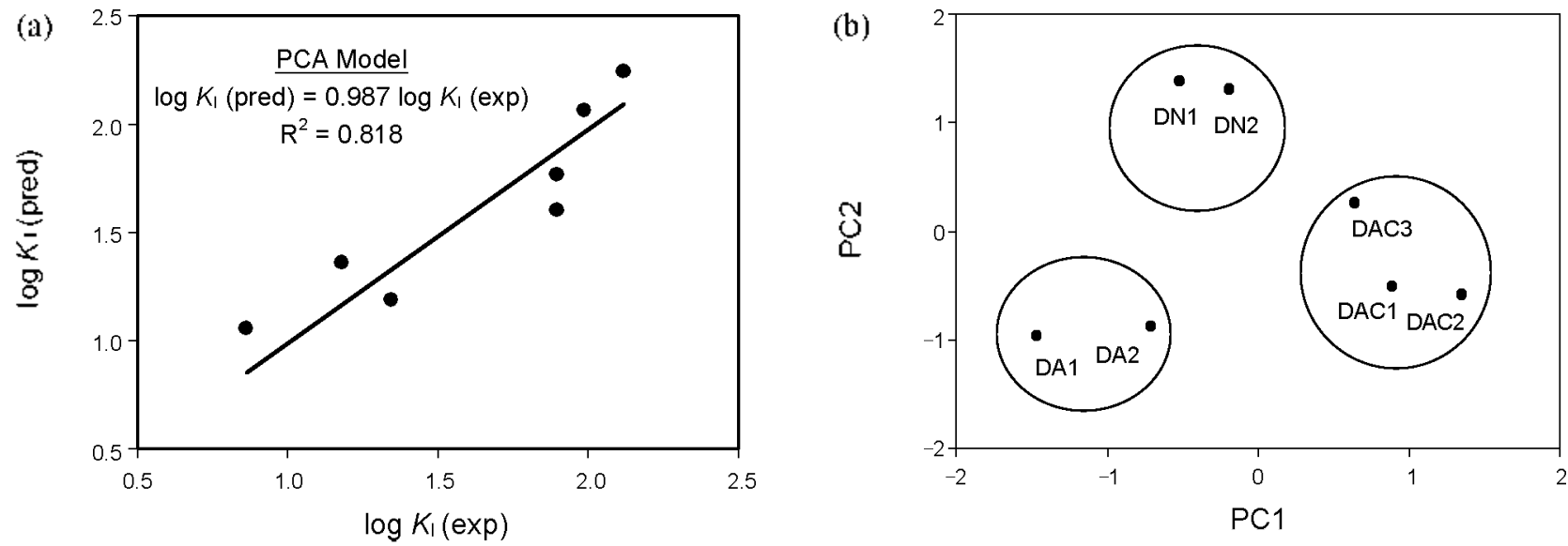

Figure 4. (a) Predicted $\log K_{\mathrm{l}}$ by PCA methods against experimental $\log K_{\mathrm{l}}$ (b) Plot of factor 2 or principal component 2 (PC2) against PC 1.

titive (DA1) and uncompeteive (DAC2) inhibitors that shows DAC2 has larger size descriptors.

Data reduction or principal component analýsis (PCA) was used to classifying the descriptors. The descriptors reduced to two factors or principal components (PCs). Table + shows that
$88 \%$ of variation can be described by these two factors. Table 5 has listed two factors obtained by data reduction (PCA). Correlation between experimental $\log K_{\mathrm{I}}$ and predicted $\log K_{\mathrm{I}}$ by PCA was shown in Fig. 4a. Variation of $\mathrm{PCl}$ ys $\mathrm{PC} 2$ also depicted in Fig. 4 b. It is seen that functional groups properly was distingui 
Table 5. Rotated component matri. ${ }^{\sigma}$ of reduced 43 descriptors into 2 factors

\begin{tabular}{|c|c|c|}
\hline \multicolumn{3}{|c|}{ Components } \\
\hline Symbol & Size & Electrotopological \\
\hline MW & 0.99895 & \\
\hline AMW & 0.86950 & \\
\hline Sv & 0.99679 & \\
\hline $\mathrm{Se}$ & 0.99653 & \\
\hline Sp & 0.99605 & \\
\hline Ss & 0.99465 & \\
\hline $\mathrm{Mv}$ & 0.87638 & \\
\hline $\mathrm{Me}$ & 0.79828 & \\
\hline $\mathrm{Mp}$ & 0.89242 & \\
\hline Ms & 0.66905 & 0.534 \\
\hline nAT & 0.99506 & \\
\hline $\mathrm{nSK}$ & 0.99884 & \\
\hline $\mathrm{nBT}$ & 0.99526 & \\
\hline $1 \mathrm{BO}$ & 0.99852 & \\
\hline $1 \mathrm{BM}$ & 0.98741 & \\
\hline SCBO & 0.99858 & \\
\hline nCIC & 0.99279 & \\
\hline nCIR & $0.97+18$ & \\
\hline $\mathrm{RBN}$ & 0.58911 & -0.754 \\
\hline $\mathrm{RBF}$ & & -0.914 \\
\hline $\mathrm{nDB}$ & 0.94786 & \\
\hline $\mathrm{nAB}$ & 0.9675 & \\
\hline $\mathrm{n} \mathrm{H}$ & 0.94204 & \\
\hline $\mathrm{nC}$ & 0.99575 & \\
\hline 10 & 0.97509 & \\
\hline $\mathrm{nR} 06$ & 0.97691 & \\
\hline$n \mathrm{Bnz}$ & 0.94692 & \\
\hline $\mathrm{Ui}$ & 0.94832 & \\
\hline Hy & -0.76814 & -0.629 \\
\hline $\mathrm{MR}$ & 0.99618 & \\
\hline PSA & 0.88353 & \\
\hline MLOGP & & 0.756 \\
\hline AROM & 0.95491 & \\
\hline HOMT & & -0.895 \\
\hline $\mathrm{SPH}$ & & 0.692 \\
\hline ASP & & -0.556 \\
\hline FDI & & 0.779 \\
\hline $\mathrm{L} / \mathrm{Bw}$ & & -0.81 \\
\hline Mux & & \\
\hline Muv & 0.51188 & 0.832 \\
\hline Muz & -0.61049 & 0.683 \\
\hline Mutot & & 0.832 \\
\hline HE & -0.96838 & \\
\hline
\end{tabular}

${ }^{a}$ Extraction Method: Principal Component Analysis. Rotation Method: Varimax with Kaiser Nomalization. Rotation converged in 3 iterations.

shed from each other by a circle.

Kinetic results showed diacids have higher inhibition constant relative to others. DAl and $\mathrm{DNI}$ are competitive and others ligands are uncompetitive. In addition. docking process was performed on the optimized ADA show that DAl and DN1 mostly were distributed around active site (Fig. 2). Autodock could not distinguish between noncompetetive and uncompetitive inhibition. It only searches and finds binding sites as well as estimates binding free energy. On the other hand, kind of inhibition is related to position of binding. Since. it will be distinguished from experimental data. Autodock only support some experimental results specially difference between competitive and other two inhibitions i.e. noncompetitive and uncompeti-
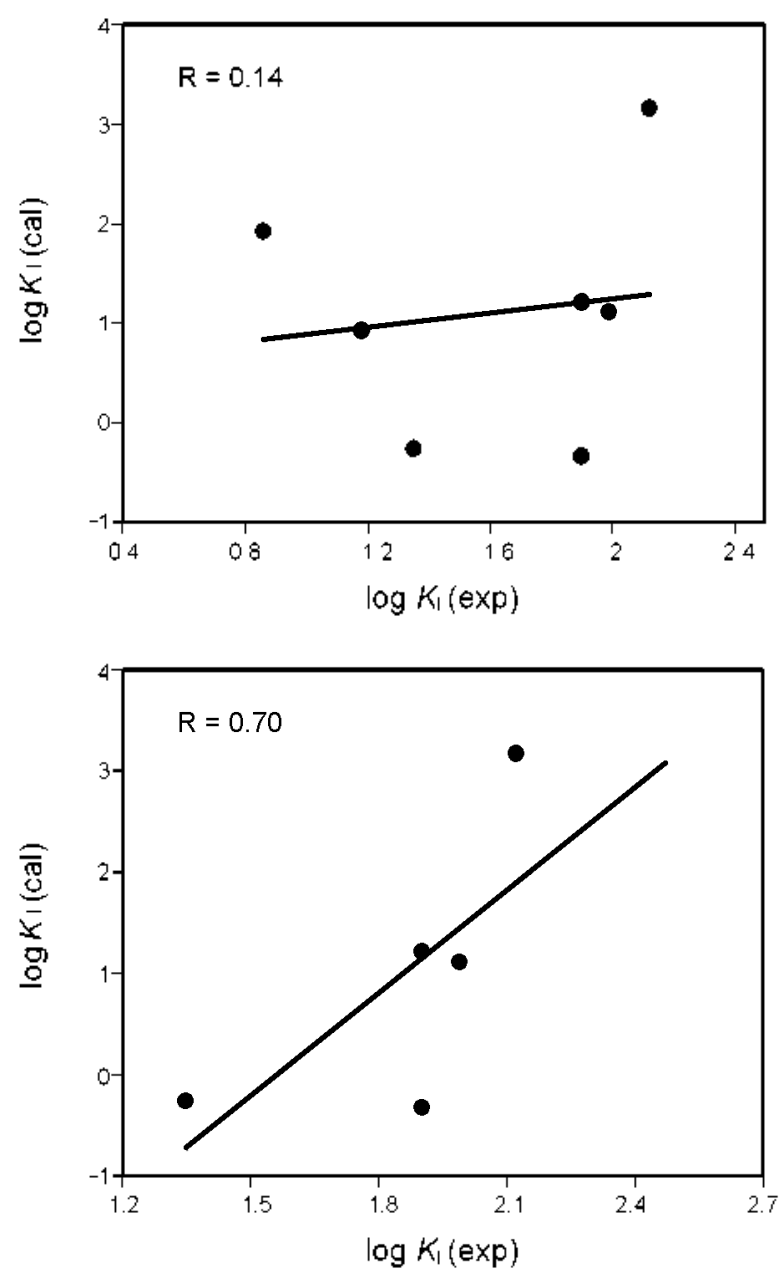

Figure 5. Calculated $\log K_{1}$ (cal) by Autodock against experimental $\log K_{1}(\mathrm{exp})$ for total ligands (up) and uncompetitive ligands (down).

tive. Because, in the former case, inhibitor binds to the actives site. while in the last two inhibitors. ligand can be located in the other sites. Computational docking usually gives a new aspect or confirmation for some experimental results. In here by docking studies we support difference between conpetitive and uncompetitive inlibitors by difference between their binding sites. Thus. they are consistence with competitive inhibition behavior. They are belongs to different groups. diamine and dinitro compounds. but they have a common property. i.e. smaller size relative to other ligands so that they can easily enter to the active site and compete with substrate. Other ligands. specially. diacids have larger size and may could not enter to active site. Thus. they are distributed in the other sites. Larger molecules select different binding sites. Equations 1, 2 also show that. competitive ligands bind only to ADA and uncompetitive ones bind to $\mathrm{ADA}$-substrate complex. It may be also related to higher degree of freedom due to ligher number of torsion angle for larger molecules. On the other hand. the active site of ADA (His17, Asp 19. Gly 184. Glu2 17. His238. 247 Asp295 and Asp296) has relatively negative charge and also partial charge of oxygen on diacid and dinitro compounds are negative. Thus. the repulsion of charge on diacids and active site anino acids, prohibit the interaction of these ligands and active site. Therefore. diacids 
could not bind to active site due to their size and charge.

Correlation between calculated data by docking and experimental inhibition constant $\left(\log K_{\mathrm{I}}\right)$ for all inhibitors and uncompetitive inhibitors were obtained and has depicted in Fig. 4. This figure shows that correlation of uncompetitive inhibitor is better than all of inhibitors. This difference is due to difference in the nature of inhibition and binding sites in competitive and uncompetitive inhibitors (Fig. l and 2).

The experimental $\log K_{\mathrm{I}}$ and calculated values of descriptors as well as correlation coefficient $(R)$ between experimental $\log K_{\mathrm{I}}$ and these calculated parameters (descriptors), for total $(R 7)$ and uncompetitive $(R 5)$ inhibitors were reported in the last two rows of this Table 4.

Correlation between $\log K_{\mathrm{I}}$ and majority of descriptors is positive. Namely, values of $\log K_{\mathrm{I}}$ increase by enhancing the descriptors values. Other descriptors. including unsaturation index (Ui), hydrophobicity (MLogP), hy'dration factor (Hy) spherocity (SPH). folding degree index (FDI). dipole moment (mux, muy, muz and mutot). and Hydration energy ( $\mathrm{HE}$ ) have negative correlation.

In addition, the most of descriptors such as; constitutional descriptors (1-27) are size dependent. while another group ( $\mathrm{SPH}$. ASP, L/Bw and FDI) are shape or geometry dependent. Third group (MogP. Hy. HE) and fourth group (AROM and HOMT) are hydrophobicity and aromaticity dependent, respectively. Finally. some of them such as dipole moment depend on more than two properties. In here. dipole moment depends on the size and electrical properties (electrotopological).

Correlation between $\log K_{\mathrm{I}}$ and size dependent descriptors is positive so that the larger molecules have more $\log K_{\mathrm{I}}$. Also. aspherocity (ASP) and length to breath ratio (L/Bw). (two geometrical or shape dependent descriptors). have positive correlation. and the other two shape dependent factors, spherocity (SPH) and folding degree index (FDI) have negative correlation. Thus, it seems that more spherical molecules and more compact, have lower inhibition constant.

The third group of descriptors related to hydrophobicity. Hydrophobicity factor (MlogP). hydration energy (HE) and hydration factor ( $\mathrm{Hy}$ ) have direct correlation with $\log K_{\mathrm{I}}$. More positive hydration energy corresponds to higher MlogP and $\mathrm{Hy}$ and less tendency for hýdration.

Fourth group, are electronic, size and shape dependent dipole moment. which have negative correlation with $\log K_{\mathrm{I}}$. The most correlated descriptor which obtained from stepwise selection. is the number of hydrogen. This descriptor increases the $\log K_{\mathrm{I}}$.

Data reduction by principal components analysis was used for classification and reduction of descriptors to a few number of principal components (PCs). Table + listed only $\mathrm{PCl}$ and PC2 that shows $88 \%$ of variation can be described by these two factors. Table 5 has listed two factors obtained by data reduction (PCA). The first factor is mostly related to size and the other is related to electrical and geometrical properties so that it is called as electrotopological descriptors which is common property between electronic and shape properties. For example hydration and shape are electronic and SPH. ASP, L/Bw; FDI, are shape descriptors. when two new reduced descriptors were studied by multiple linear regression the following equation was obtained:

$$
\begin{aligned}
\log K_{\mathrm{I}}= & 0.346 \text { (Size factor) } \\
& -0.271 \text { (electrotopological factor })+1.6
\end{aligned}
$$

It is shown that the $\log K_{\mathrm{I}}$ increases and decreases with size and electrotopological factors respectively: Thus. larger molecule has higher inhibition constant. We also reported previously that $K_{\mathrm{I}}$ for non-nucleosides inhibitors increases by size and shape. ${ }^{20}$ Fig. ta shows the correlation between predicted by equation (3) and experimental values of $\log K_{\text {I. }}$. Fig. tb shows the variation of factor $2(\mathrm{PC} 2)$ versus factor $1(\mathrm{PC} 1)$. It indicates that each of three groups which have similar properties were surrounded by a circle. It means that. we can distinguish the similar groups only from descriptors without using any other techniques.

\section{Conclusion}

Effect of different organic aromatic ligands on the structure and activity of ADA was studied by experimental and computational methods. All of ligands showed inhibition behavior, some of them were mixed of competitive and non-competitive (one of diaamines. and one of dinitros) and the others (nostly diacids) were uncompetitive inlubitors. There is a logical correlation between experimental and calculated inhibition constant for uncompetitive inhibitors. Calculated binding energy and binding site of ligands showed that competitive inhibitors are relatively small ligands. and is distributed around active site of enzy me with higher cluster rank. The experimental results also showed that competitive inhibitors bind to the active site. whereas uncompetitive ligands are larger and have different binding site in accord to experimental results. Results of structureproperty relationship showed that, larger. more hydrophobe, less spherical and more aromatic ligands have higher inhibition constant.

Acknowledgments. Financial support of Danghan University of Basic Science is acknowledged. Author is also grateful to Dr Hossein Beluniafar for providing the needed ligands.

\section{References}

1. Frick, L.; Wolfenden, R.: Smal, E.: Baker D. C. Biochemismy $1986,8,1616$

2. Chechik, B. E.: Schreder, W. P.; Minowada, J. J. Inmmol. 1981 , 126, 1003 .

3. Herschfeld, M. S.; Mitchell, B. S. In the Metabolic and Molecular Basis of Inherted Disease: Scriver C. R.; Bealudet A. L.: Sly W. S.; Valle, D., Eds.; McGraw-Hill: New York, 1995; p 1725.

4. Herschfield, M. S. Semin. Hentatol. 1998, 35, 291.

5. Phillis. J. W. Wh. P. H. Biol Repiod Prog Nemobiol 1981, 16, 187.

6. Cronstein, B. N. J. Appl. Physiol. 1994, 76, 5

7. Ohta, A Nanure 2001, $11+916$.

8. Rudolphi, K. A.; Schubert, P.; Parkinson, F. E.: Fredholm, B. B. Trends Phamtacol. Sci. 1992, 13, 439

9. Marquardt, D. L.: Gruber, H. E.; Wasserman, S. I. Proc. Natl. Acad. Sci. US.4. 1984, 81,6192.

10. Franco, R; Valenzuela, A.; Luis, C; Blanco, T. Immunol. Rev: $1998,161,27$ 
11. Baker, D. C.: Hanvey, J. C.: Hawkins, L. D.: Muphy, T. Biochem. Phamacol 1981, 30, 1159

12. Saboury, A. A.: Divsalar, A.: Ataie, G.: Moosavi-Movahedi, A. A. J. Biochem. Mol. Biol. 2002, 35, 302.

13. Saboury, A. A.: Divsalar, A.: Ataie, G.: Amanlou, M.: MoosaviMovahedi, A. A. Acta Biochem. Pol 2003, 50, 849

14. Saboury, A. A.: Bagheri, S.; Ataie, G.; Amanlou, M.; MoosaviMovahedi, A. A.; Hakimelahi, G. H.; Cristalli, G. S. Chem. Phorm. Bull. 2004, 52, 1179 .

15. Ataie, G.; Safarian, S.; Divsalar, A.; Saboury, A. A.: MoosaviMovahedi, A. A.: Ranjbar, B.: Cristalli, G.: Mardanian, S. J. Enz. Inhib. Med Chem. 2004, 19,71.

16. Sabourv, A. A. Bagheri, S : Ataie, G.: Moosavi-Movahedi, A. A. Hakimelahi, G. H.; Cristalli, G.; Mardanian, S. A. Asian J. Chem. $2005,17,233$

17. Saboury, A. A. J. Thermal. Anal. Cal. 2003, 72,93.

18. Ataie, G.: Moosavi-Movahedi, A. A.: Sabour, A. A.: Hakimelahi, G. H.; Hwu, T. R. Tsav, S. C. Int. J. Biol. M facromol 2000, 27, 29

19. Moosavi-Movahedi. A. A.: Safarian1. S.: Hakinelahi. G. H.: Ataei. G.; Ajlow, D.: Panjehpour, S.; Riahi, S.: Mousavi, M F.: Mardanyan,
S. Nucleos. Nucleot. Nucl. 2004. 3.613.

20. Ajloo, D.; Saboury, A. A.; Haghi-Asli, N.: Atale-Iafani, G.; MossaviMovahedi, A. A.: Ahmadi, M.; Mahnam, K.: Namaki, S. J. Enz. Inhib. Med. Chem 2007, 22,395.

21. Ajloo, D; Taghizadeh, E.; Saboury, A. A.; Bazyari, E.; Mahnam, K. Iht J. Biol Macromol 2008, 13, 158 .

22. Banilhashemi. A. Behniafar. H. Pohm. Int. 2003, 52,1136

23. Behniafar, H.; Banihashemi, A. Polm. Int. 2004, $53,2020$.

24. Kaplan, N. O. Meth Enzmol; Academic Press: New York, 1955 ; Vol. 2, p 473

25. Todeschini, R.: Consonni, V; Mauri, A.; Pavan, M. Dragon-3.0, 2003.

26. Todeschini, R: Consonni, V th the Iarious Holecular Descriptors Handbook of Holectiar Descriptors: Wiley-VCH: Weinheim, Germany, 2000 .

27. Morris G. M.; Goodsell, D. S.: Halliday, R. S.; Huley, R.; Hart, W. E.; Belew, R. K.; Olson, A. J. J. Conput, Chem. $1998,19,1639$.

28. Segel, I. H. Enzme Kinetics: Behavior and Analvis of Rapid Equilibium and Steady-state Enzzme Systems; Wiley: New York, 1993 